\title{
COMMENTARY
}

\section{Type 1 and type 2 diabetes-chalk and cheese?}

\author{
K. R. Owen • M. I. McCarthy
}

Received: 17 May 2009 /Accepted: 10 July 2009 /Published online: 1 August 2009

(C) Springer-Verlag 2009

Keywords Genetics of type 1 diabetes - Genetics of type 2 diabetes $\cdot$ Latent autoimmune diabetes of adulthood

\section{Abbreviation \\ LADA Latent autoimmune diabetes of adulthood}

The conventional wisdom is that the two major subtypes of diabetes, type 1 and type 2, represent distinct conditions with widely different pathophysiologies. However, the growing recognition that a substantial proportion of people with diabetes have features of both type 1 and type 2 diabetes [1] indicates that it may not always be possible to assign each patient to a neat diagnostic box. Instead, we should probably be thinking more in terms of a spectrum of disease, with 'typical' type 1 diabetes at one extreme and 'typical' type 2 diabetes at the other. Indeed, some go further and try to explain the clinical overlap, together with the growing incidence of all types of diabetes, by proposing

\footnotetext{
K. R. Owen • M. I. McCarthy $(\bowtie)$

Oxford Centre for Diabetes, Endocrinology and Metabolism,

University of Oxford, Churchill Hospital,

Old Road, Headington,

Oxford OX3 7LJ, UK

e-mail: mark.mccarthy@drl.ox.ac.uk

K. R. Owen • M. I. McCarthy

Oxford NIHR Biomedical Research Centre, Churchill Hospital,

Oxford, UK

M. I. McCarthy

Wellcome Trust Centre for Human Genetics,

University of Oxford,

Oxford, UK
}

that both major forms of diabetes share common aetiological factors. One widely cited example is the 'accelerator hypothesis' [2], which holds that increasing rates of obesity and insulin resistance contribute to the development of type 1 and type 2 diabetes by driving a more rapid decline in beta cell function, irrespective of the nature of the original islet insult.

By defining fundamental pathophysiological processes underlying individual susceptibility to disease, genetic analyses have the potential to inform debates such as these. With the recent crop of genome-wide association studies taking the tally of loci confidently implicated in the pathogenesis of multifactorial forms of diabetes [35] to more than 60 , it has become possible to use these to explore the 'aetiological architecture' of diabetes. A number of recent papers describe attempts to do just this [6-14].

In this issue of Diabetologia, Raj and colleagues take a panel of variants known to influence individual risk of type 2 diabetes and examine their effects on predisposition to type 1 diabetes [12]. Since the effects of these variants on type 2 diabetes risk are quite modest (odds ratios of 1.1 to 1.4 per allele), an important feature of this study was the use of large sample collections (more than 25,000 individuals). This allowed the authors to be confident that they had not missed small, but nonetheless informative, effects of these variants on risk of type 1 diabetes. Apart from a modest association between the Pro12Ala variant in the PPARG gene and type 1 diabetes, there was no evidence that these type 2 diabetes risk variants impact on susceptibility to type 1 diabetes. The authors conclude that this argues strongly against pathophysiological mechanisms common to both major forms of diabetes. 
These findings seem hard to reconcile with hypotheses that invoke aetiological mechanisms common to type 1 and type 2 diabetes. Perhaps, though, the paper by Raj and colleagues is not quite the 'knockout' blow that it first appears. One limitation is that the panel of 12 variants examined explains only a minority (probably between 5\% and $10 \%$ ) of the inherited susceptibility to type 2 diabetes [4]. As additional type 2 diabetes susceptibility loci emerge from large-scale genotyping and re-sequencing studies, it is conceivable that some of these might highlight pathways that, in contrast to those uncovered so far, are shared by type 1 and type 2 diabetes.

It is also worth noting that most of the type 2 diabetes variants tested by Raj and colleagues are thought to operate by effects on beta cell function and/or mass. Of those tested, only the variants in PPARG and FTO influence type 2 diabetes predisposition through effects on insulin action or obesity [15]. It is these, then, which represent the most pertinent tests of the accelerator hypothesis. Interestingly, Raj and colleagues did find that the Ala12 variant allele in PPARG, which has long been known to be protective against type 2 diabetes [16], also appears to have a similar protective effect on type 1 diabetes, corroborating an observation previously reported in Danish parent-offspring trios [17]. Since one of the downstream effects of the Ala allele is to improve insulin sensitivity [18], this observation could be considered to support the 'accelerator hypothesis'. However, failure to detect similar effects on type 1 diabetes risk at FTO [11, 13] is difficult to square with this interpretation, and Raj and colleagues propose instead that the relationship between Pro12Ala and type 1 diabetes risk is mediated through the known actions of peroxisome proliferator-activated receptor gamma on inflammation [19].

If we accept, with these qualifications, that the results from Raj et al. argue against a great deal of overlap in the pathogenesis of type 1 and type 2 diabetes, what should we make of conditions such as latent autoimmune diabetes of adulthood (LADA), which manifestly have elements of both [20]? The diagnosis of LADA typically rests on the presence of islet autoantibodies in a diabetic individual for whom there is no absolute and immediate requirement for long-term insulin therapy. Several difficulties complicate the interpretation of research in this area, including absence of a consistent definition [21] and diagnostic reliance on a measure (i.e. beta cell autoantibodies) for which assays are not always standardised [22] and for which there is a non-trivial background level of positivity in the non-diabetic population. The clinical significance of autoantibody-positivity in otherwise healthy individuals is unclear, but it does mean that the mere presence of autoantibodies cannot be taken as certain proof that islet autoimmunity was causal for the diabetic phenotype in any given patient. Nonetheless, clinical studies do define a consistent phenotype of individuals who show fewer signs of insulin resistance than antibodynegative (type 2 diabetic) patients and who progress to insulin therapy more rapidly [23].

Here again, genetic studies are providing important aetiological clues. A series of papers over the past decade has shown that individuals with LADA share the HLA, insulin gene and PTPN22 variant associations that are characteristic of type 1 diabetes [8, 9, 12, 24]. These findings establish that the islet autoimmunity of LADA is causal (rather than secondary to exposure to antigens released from islet cells destroyed by other processes). They also indicate that LADA and type 1 diabetes have common aetiological mechanisms. The widely accepted view is that LADA is a 'forme fruste' of type 1 diabetes, representing a more indolent course of autoimmune beta cell destruction occurring in individuals with less extreme genetic predisposition and/or more restricted exposure to the relevant environmental triggers.

A recent paper appears to challenge this view [7]. Cervin and colleagues showed that variants in the TCF7L2 gene (which have the largest effect on type 2 diabetes susceptibility of any common variant yet identified) are also strongly associated with LADA. This finding would seem to point towards shared determinants of type 2 diabetes and LADA, reigniting the notion of overlap between the development of type 2 diabetes and propensity to autoimmune diabetes.

Could inflammation be one process that unites autoimmune and non-autoimmune forms of diabetes? The association between PPARG variants and type 1 diabetes [13, 17] would be consistent with such an idea. Moreover, type 2 diabetes is certainly characterised by raised inflammatory markers [25]. However, the observation that variants known to influence inflammatory and immune processes (including many of the variants implicated in type 1 diabetes susceptibility) show no evident relationship to type 2 diabetes risk argues strongly against this view [26]. Besides, if the TCF7L2 association with LADA betrays overlap with autoimmune disease, the same TCF7L2 variants should surely influence type 1 diabetes risk, a hypothesis which has been comprehensively refuted $[10,13]$.

There may, of course, be other, more innocent, explanations to the findings of Cervin and colleagues. One relatively trivial solution relates to the high background level of autoantibodies in the population being tested. Given a level of GADA positivity in their controls of around 4\% [24], any definition assigning all type 2 diabetes individuals who also happen to be GADA-positive $(9 \%$ overall) to the category of LADA could be expected to result in a heterogeneous grouping that includes 'true 
LADA' and 'type 2 diabetes with incidental islet autoimmunity' and therefore has genetic similarities to both type 1 and type 2 diabetes.

The second possible explanation builds on the high prevalence of type 2 diabetes and islet autoimmunity. Both can result in progressive beta cell failure, albeit through different mechanisms, and once the depletion in beta cell function reaches a certain point, diabetes ensues. Many individuals will have inherited an enhanced genetic predisposition to both autoimmune and to type 2 diabetes. Of those, many will have encountered the relevant environmental triggers (whatever those may be). It is not hard to imagine therefore that, for many individuals, the route to beta cell failure involves the additive influence of autoimmune- and type 2 diabetes-related mechanisms. Several of the type 2 diabetes susceptibility loci, for example, may influence beta cell mass, through effects on development, replication or senescence [4], rendering those unlucky enough to have inherited a large number of such susceptibility alleles especially vulnerable to any other process that compromises beta cell performance. The groups of relatively early-onset LADA cases that tend to be recruited to genetic studies are likely to be enriched for both an autoimmune diathesis and type 2 diabetes predisposition, since the combined action of indolent islet autoimmunity and subtle (type 2 diabetes-related) beta cell failure may generate diabetes some years earlier than either acting alone. Little surprise then, that type 2 diabetes susceptibility alleles can be detected in such studies, although the size of currently available LADA collections (a few hundred cases at the most) makes it difficult to demonstrate this for variants with effect-sizes smaller than those in TCF7L2.

What do all these studies tell us about the 'aetiological architecture' of the different diabetes subtypes? Because autoimmune and type 2 diabetes are common and because they converge towards progressive beta cell failure, they may jointly contribute to the development of diabetes in a proportion of patients. In these individuals it would be entirely appropriate to speak of shared aetiology and overlap. However, there seems little from these genetic studies to disturb the conventional view that type 1 diabetes and LADA are diseases primarily driven by the autoimmune destruction of pancreatic beta cells and that type 2 diabetes is a condition of progressive beta cell failure attributable to entirely distinct (but as yet uncertain) mechanisms.

Acknowledgements K. R. Owen is funded by a National Institute for Health Research (NIHR) clinician scientist award.

Duality of interest The authors declare that there is no duality of interest associated with this manuscript.

\section{References}

1. Pozzilli P, Buzzetti R (2007) A new expression of diabetes: double diabetes. Trends Endocrinol Metab 18:52-57

2. Wilkin TJ (2001) The accelerator hypothesis: weight gain as the missing link between type I and type II diabetes. Diabetologia 44:914-922

3. Barrett JC, Clayton DG, Concannon P et al (2009) Genome-wide association study and meta-analysis find that over 40 loci affect risk of type 1 diabetes. Nat Genet. doi:10.1038/ng.381

4. Prokopenko I, McCarthy MI, Lindgren CM (2008) Type 2 diabetes: new genes, new understanding. Trends Genet 24:613621

5. Zeggini E, Scott LJ, Saxena R et al (2008) Meta-analysis of genome-wide association data and large-scale replication identifies additional susceptibility loci for type 2 diabetes. Nat Genet 40:638-645

6. Bakhtadze E, Cervin C, Lindholm E et al (2008) Common variants in the TCF7L2 gene help to differentiate autoimmune from non-autoimmune diabetes in young (15-34 years) but not in middle-aged (40-59 years) diabetic patients. Diabetologia 51:2224-2232

7. Cervin C, Lyssenko V, Bakhtadze E et al (2008) Genetic similarities between latent autoimmune diabetes in adults, type 1 diabetes, and type 2 diabetes. Diabetes 57:1433-1437

8. Desai M, Zeggini E, Horton VA et al (2006) The variable number of tandem repeats upstream of the insulin gene is a susceptibility locus for latent autoimmune diabetes in adults. Diabetes 55:18901894

9. Desai M, Zeggini E, Horton VA et al (2007) An association analysis of the HLA gene region in latent autoimmune diabetes in adults. Diabetologia 50:68-73

10. Field SF, Howson JM, Smyth DJ, Walker NM, Dunger DB, Todd JA (2007) Analysis of the type 2 diabetes gene, TCF7L2, in 13,795 type 1 diabetes cases and control subjects. Diabetologia 50:212-213

11. Field SF, Howson JM, Walker NM, Dunger DB, Todd JA (2007) Analysis of the obesity gene FTO in 14,803 type 1 diabetes cases and controls. Diabetologia 50:2218-2220

12. Petrone A, Suraci C, Capizzi M et al (2008) The protein tyrosine phosphatase nonreceptor 22 (PTPN22) is associated with high GAD antibody titer in latent autoimmune diabetes in adults: Non Insulin Requiring Autoimmune Diabetes (NIRAD) Study 3. Diabetes Care 31:534-538

13. Raj SM, Howson JMM, Walker NM et al (2009) No association of multiple type 2 diabetes loci with type 1 diabetes. Diabetologia. doi:10.1007/s00125-009-1391-y

14. Qu HQ, Grant SF, Bradfield JP et al (2008) Association analysis of type 2 diabetes loci in type 1 diabetes. Diabetes 57:1983-1986

15. Florez JC (2008) Newly identified loci highlight beta cell dysfunction as a key cause of type 2 diabetes: where are the insulin resistance genes? Diabetologia 51:1100-1110

16. Altshuler D, Hirschhorn JN, Klannemark M et al (2000) The common PPARgamma Pro12Ala polymorphism is associated with decreased risk of type 2 diabetes. Nat Genet 26:76-80

17. Johansen A, Jensen DP, Bergholdt R et al (2006) IRS1, KCNJ11, PPARgamma2 and HNF-1alpha: do amino acid polymorphisms in these candidate genes support a shared aetiology between type 1 and type 2 diabetes? Diabetes Obes Metab 8:75-82

18. Heikkinen S, Argmann C, Feige JN et al (2009) The Pro12Ala PPARgamma2 variant determines metabolism at the geneenvironment interface. Cell Metab 9:88-98

19. Szeles L, Torocsik D, Nagy L (2007) PPARgamma in immunity and inflammation: cell types and diseases. Biochim Biophys Acta 1771:1014-1030 
20. Owen KR, Stride A, Ellard S, Hattersley AT (2003) Etiological investigation of diabetes in young adults presenting with apparent type 2 diabetes. Diabetes Care 26:2088-2093

21. No authors listed (2009) Diagnosis and classification of diabetes mellitus. Diabetes Care 32(Suppl 1):S62-S67

22. Törn C, Mueller PW, Schlosser M, Bonifacio E, Bingley PJ, Laboratories P (2008) Diabetes Antibody Standardization Program: evaluation of assays for autoantibodies to glutamic acid decarboxylase and islet antigen-2. Diabetologia 51:846852

23. Turner R, Stratton I, Horton V et al (1997) UKPDS 25: autoantibodies to islet-cell cytoplasm and glutamic acid decarboxylase for prediction of insulin requirement in type 2 diabetes. UK Prospective Diabetes Study Group. Lancet 350:1288-1293

24. Tuomi T, Carlsson A, Li H et al (1999) Clinical and genetic characteristics of type 2 diabetes with and without GAD antibodies. Diabetes 48:150-157

25. Greenfield JR, Campbell LV (2006) Relationship between inflammation, insulin resistance and type 2 diabetes: 'cause or effect'? Curr Diabetes Rev 2:195-211

26. Rafiq S, Melzer D, Weedon MN et al (2008) Gene variants influencing measures of inflammation or predisposing to autoimmune and inflammatory diseases are not associated with the risk of type 2 diabetes. Diabetologia 51:2205-2213 